PROCEEDINGS OF THE

AMERICAN MATHEMATICAL SOCIETY

Volume 139, Number 7, July 2011, Pages 2631-2645

S 0002-9939(2010)10698-6

Article electronically published on December 22, 2010

\title{
TURAEV GENUS, KNOT SIGNATURE, AND THE KNOT HOMOLOGY CONCORDANCE INVARIANTS
}

\author{
OLIVER T. DASBACH AND ADAM M. LOWRANCE
}

(Communicated by Daniel Ruberman)

\begin{abstract}
We give bounds on knot signature, the Ozsváth-Szabó $\tau$ invariant, and the Rasmussen $s$ invariant in terms of the Turaev genus of the knot.
\end{abstract}

\section{INTRODUCTION}

Alternating knots have particularly simple reduced Khovanov homology and knot Floer homology. Lee Lee05 showed that the reduced Khovanov homology of an alternating knot $K$ is fully determined by its Jones polynomial $V_{K}(q)$ and its signature $\sigma(K)$. Analogously, Ozsváth and Szabó OS03a proved that the knot Floer homology of an alternating knot $K$ is determined by its Alexander polynomial $\Delta_{K}(t)$ and its signature $\sigma(K)$. Furthermore, if $K$ is an alternating knot, then the Ozsváth-Szabó $\tau$ invariant OS03b], the Rasmussen $s$ invariant Ras04, and the signature of $K$ satisfy

$$
2 \tau(K)=s(K)=-\sigma(K) .
$$

Note that it took some effort to show that in general $2 \tau(K)$ and $s(K)$ are not equal $\mathrm{HO} 08$.

To compute the signature, if $D$ is a reduced alternating diagram of a knot $K$, Traczyk Tra04] proved that

$$
\begin{aligned}
\sigma(K) & =s_{A}(D)-n_{+}(D)-1 \\
& =1+n_{-}(D)-s_{B}(D),
\end{aligned}
$$

where $s_{A}(D)$ and $s_{B}(D)$ are the number of components in the all- $A$ and all- $B$ Kauffman resolutions of $D$ respectively, and $n_{+}(D)$ and $n_{-}(D)$ are the number of positive and negative crossings in $D$ respectively. Throughout this paper we choose our sign convention for the signature such that the signature of the positive trefoil is -2 .

Our goal is to generalize the relationships between knot signature, the OzsváthSzabó $\tau$ invariant, the Rasmussen $s$ invariant, and Traczyk's combinatorial knot diagram data from equations (1.1) and (1.2) to non-alternating knots. These relationships lead to new lower bounds for the Turaev genus of a knot.

Received by the editors March 9, 2010 and, in revised form, July 6, 2010.

2010 Mathematics Subject Classification. Primary 57M25, 57M27.

The first author was partially supported by NSF-DMS 0806539 and NSF-DMS FRG 0456275.

The second author was partially supported by NSF-DMS 0739382 (VIGRE) and NSF-DMS 0602242 (VIGRE).

(C)2010 American Mathematical Society Reverts to public domain 28 years from publication 
For a given knot diagram in the plane, Turaev Tur87 constructed an embedded oriented surface $\Sigma_{D}$ on which the knot projects. In $\left[\mathrm{DFK}^{+} 08\right.$ ] it is pointed out that the knot projection is alternating on the Turaev surface and that the Turaev surface is a Heegaard surface for $S^{3}$. The precise construction of the Turaev surface is given in Section 4. The Turaev genus of a knot $g_{T}(K)$ is the minimum genus of $\Sigma_{D}$ over all diagrams of the knot. It is worth noting that the Turaev genus of a knot is zero if and only if the knot is alternating $\mathrm{DFK}^{+} 08$. We will relate the Turaev genus of a knot $K$ with $\sigma(K), \tau(K)$ and $s(K)$ in the following:

Theorem 1.1. Let $K$ be a knot. Then

$$
\begin{aligned}
\left|\tau(K)+\frac{\sigma(K)}{2}\right| & \leq g_{T}(K), \\
\frac{|s(K)+\sigma(K)|}{2} & \leq g_{T}(K), \text { and } \\
\left|\tau(K)-\frac{s(K)}{2}\right| & \leq g_{T}(K) .
\end{aligned}
$$

For alternating knots, i.e. when $g_{T}(K)=0$, those inequalities reflect the results of Oszváth, Szabó, and Rasmussen.

Abe Abe09, using work of Livingston [Liv04, has shown that the three quantities on the left in Theorem 1.1 are also lower bounds for the alternation number of a knot, which is the minimum Gordian distance between a given knot and any alternating knot. Examining how the Turaev genus of a knot compares to its alternation number remains an interesting open problem.

The paper is organized as follows. In Section 2 we review the constructions of the Ozsváth-Szabó $\tau$ invariant and the Rasmussen $s$ invariant. In Section 3 , we show a relationship between the spanning tree complexes for reduced Khovanov homology and knot Floer homology. Section 4 is a review of the construction of the Turaev surface and its relationship to the spanning tree complexes. Finally, we show how knot signature fits into the picture in Section 5. In Section 6] we compute the bounds of Theorem 1.1 for knots obtained as the closure of 3-braids.

\section{KNOT HOMOLOGY CONCORDANCE INVARIANTS}

In this section, we recall the definitions of the Ozsváth-Szabó $\tau$ invariant OS03b and the Rasmussen $s$ invariant [Ras04].

2.1. Ozsváth-Szabó $\tau$ invariant. Heegaard Floer homology is an invariant for closed 3-manifolds defined by Ozsváth and Szabó in OS04c and OS04b. The Heegaard Floer package gives rise to a concordance invariant, called the OzsváthSzabó $\tau$ invariant, whose construction is given below.

Suppose $(\Sigma, \alpha, \beta, w, z)$ is a Heegaard diagram subordinate to the knot $K$ in $S^{3}$. This means $\Sigma$ is a genus $g$ surface and both $\alpha=\left\{\alpha_{1}, \ldots, \alpha_{g}\right\}$ and $\beta=\left\{\beta_{1}, \ldots, \beta_{g}\right\}$ are $g$-tuples of homologically linearly independent, pairwise disjoint, simple closed curves in $\Sigma$. Also, $w$ and $z$ are points in the complement of the $\alpha$ and $\beta$ curves in $\Sigma$ lying in a neighborhood of the curve $\beta_{1}$ and situated on opposite sides of $\beta_{1}$. The two sets of curves $\alpha$ and $\beta$ are boundaries of attaching disks and specify handlebodies $U_{\alpha}$ and $U_{\beta}$ both with boundary $\Sigma$ and $U_{\alpha} \cup_{\Sigma} U_{\beta} \cong S^{3}$. The knot $K$ can be isotoped onto $\Sigma$ such that it is disjoint from $\beta_{2}, \ldots, \beta_{g}$, an arc of $K$ runs from the basepoint $w$ to the basepoint $z$, and this arc intersects $\beta_{1}$ once transversely. 
Denote the $g$-fold symmetric product of $\Sigma$ by $\operatorname{Sym}^{g}(\Sigma)$ and consider the two embedded tori $\mathbb{T}_{\alpha}=\alpha_{1} \times \cdots \times \alpha_{g}$ and $\mathbb{T}_{\beta}=\beta_{1} \times \cdots \times \beta_{g}$. Let $\widehat{C F}\left(S^{3}\right)$ denote the $\mathbb{Z}$-module generated by the intersection points of $\mathbb{T}_{\alpha}$ and $\mathbb{T}_{\beta}$. The complex $\widehat{C F}\left(S^{3}\right)$ can be endowed with a differential that counts pseudo-holomorphics disks in $\operatorname{Sym}^{g}(\Sigma)$ between intersection points of $\mathbb{T}_{\alpha}$ and $\mathbb{T}_{\beta}$. The homology of $\widehat{C F}\left(S^{3}\right)$ is denoted $\widehat{H F}\left(S^{3}\right)$ and is isomorphic to $\mathbb{Z}$ (appearing in homological grading zero).

Ozsváth and Szabó OS04a and independently Rasmussen Ras03 proved that a knot $K$ induces a filtration on the chain complex $\widehat{C F}\left(S^{3}\right)$. Define $\mathcal{F}(K, m) \subset$ $\widehat{C F}\left(S^{3}\right)$ to be the subcomplex generated by intersection points with filtration level less than or equal to $m$. There is an induced sequence of maps

$$
\imath_{K}^{m}: H_{*}(\mathcal{F}(K, m)) \rightarrow H_{*}\left(\widehat{C F}\left(S^{3}\right)\right)=\widehat{H F}\left(S^{3}\right) \cong \mathbb{Z}
$$

that are isomorphisms for all sufficiently large integers $m$. The Ozsváth-Szabó $\tau$ invariant is defined as

$$
\tau(K)=\min \left\{m \in \mathbb{Z} \mid \imath_{K}^{m} \text { is non-trivial }\right\} .
$$

By construction $\tau(K)$ is a knot invariant, and Ozsvath and Szabó OS03b showed that $\tau(K)$ depends only on the concordance class of $K$.

Also, recall that one can use the filtration $\mathcal{F}(K, m)$ to define the knot Floer homology of $K$, denoted $\widehat{H F K}(K)$, as follows. Define

$$
\widehat{H F K}(K)=\bigoplus_{m \in \mathbb{Z}} H_{*}(\mathcal{F}(K, m) / \mathcal{F}(K, m-1)) .
$$

Thus $\widehat{H F K}(K)$ is the homology of the complex $\widehat{C F K}(K)$, where $\widehat{C F K}(K)$ is generated by intersection points of $\mathbb{T}_{\alpha}$ and $\mathbb{T}_{\beta}$, but unlike in $\widehat{C F}\left(S^{3}\right)$, the differential in $\widehat{C F K}(K)$ must preserve filtration level.

2.2. The Rasmussen $s$ invariant. Khovanov homology [Kho00] is a knot invariant that categorifies the Jones polynomial. Rasmussen Ras04 used Lee's deformation of Khovanov homology Lee05] to define a concordance invariant, known as the Rasmussen $s$ invariant, whose construction is described below.

Let $D$ be a diagram of a knot $K$ with crossings labelled 1 through $k$. Each crossing of $D$ has an $A$-smoothing and a $B$-smoothing, as shown in Figure 1 , Associate to each vertex $I$ of the cube $\{A, B\}^{k}$ the collection of simple closed curves in the plane $D_{I}$ obtained by smoothing the $i$-th crossing of $D$ according to the $i$-th coordinate of $I$. To each $D_{I}$ associate the $\mathbb{Q}$-vector space $V^{\otimes|I|}$ where $V$ is free on two generators $v_{+}$and $v_{-}$and $|I|$ is the number of components in $D_{I}$. Define a bigraded $\mathbb{Q}$-vector space, known as the cube of resolutions, by

$$
C K h(D)=\bigoplus_{I \in\{A, B\}^{k}} V^{\otimes|I|}
$$

The homological grading of each summand $V^{\otimes|I|}$ is the number of $B$-smoothings in $I$ minus the number of negative crossings in $D$ (as in Figure 2).

We will investigate two different differentials on $C K h(D)$. The first, $\partial_{K h}$, is Khovanov's differential. The homology $H_{*}\left(C K h(D), \partial_{K h}\right)$ is denoted $K h(K)$. The vector space $K h(K)$ has a homological and Jones grading, and its filtered Euler characteristic is $\left(q^{1 / 2}+q^{-1 / 2}\right) V_{K}(q)$ where $V_{K}(q)$ is the Jones polynomial of $K$. (Note that we normalize the Jones grading to be half the usual grading.) The 


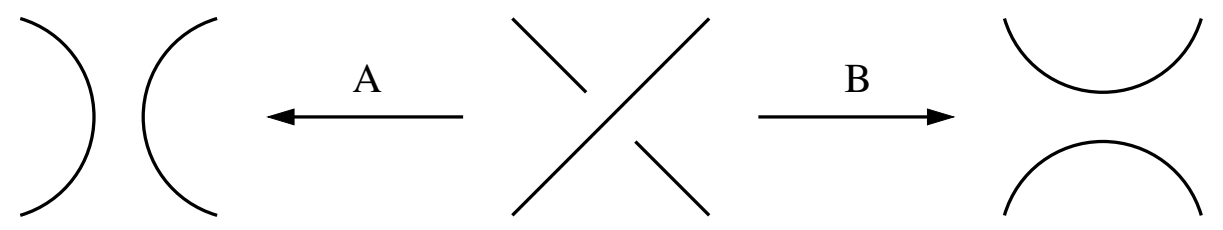

Figure 1. The $A$ and $B$ smoothings of a crossing.

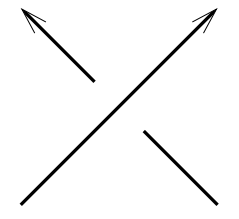

positive

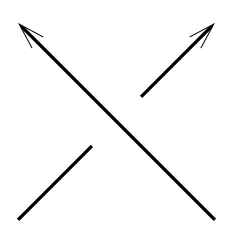

negative

Figure 2. The crossing on the left is positive, and the crossing on the right is negative.

second, $\partial_{\text {Lee }}$, is Lee's differential. The homology $K h^{\prime}(K):=H_{*}\left(C K h(D), \partial_{\text {Lee }}\right)$ is isomorphic to $\mathbb{Q} \oplus \mathbb{Q}$. Lee's differential can be written as $\partial_{\text {Lee }}=\partial_{K h}+\Phi$ where $\Phi$ increases Jones grading. The following theorem is implicit in Lee Lee05] and explicitly stated in Rasmussen Ras04.

Theorem 2.1 (Rasmussen Ras04). Let $K$ be a knot. There is a spectral sequence with $E_{2}$ term $K h(K)$ that converges to $K h^{\prime}(K)$. The $E_{2}$ and higher terms of this spectral sequence are invariants of $K$.

Lee identifies elements of $C K h(D)$ that represent the homology classes $\mathbb{Q} \oplus \mathbb{Q}$. These cycles are elements of $V^{\otimes|I|}$ where $I$ is the vertex obtained by smoothing each crossing according the orientation of the knot; i.e. if a crossing is positive, then one chooses the $A$-smoothing, and if a crossing is negative, then one chooses the $B$-smoothing. Therefore, the homological gradings of both of these cycles must be zero.

Lee's differential does not preserve the Jones grading. In order to obtain a welldefined Jones grading on Lee's homology, one must minimize over all elements in a given homology class. More specifically, if $\alpha \in H_{*}\left(C K h(D), \partial_{\text {Lee }}\right)$, then the Jones grading of $\alpha$ is the minimum Jones grading of any element $a$ of $C K h(D)$ such that $a$ represents the homology class $\alpha$.

In Ras04, Rasmussen showed that Lee's homology is supported in two Jones gradings $s_{\min }(K)$ and $s_{\max }(K)$ depending only on $K$, and moreover $s_{\max }(K)=$ $s_{\min }(K)+1$. Since our Jones grading is half of Khovanov's original Jones grading, both $s_{\min }(K)$ and $s_{\max }(K)$ are in $\mathbb{Z}+\frac{1}{2}$. The Rasmussen $s$ invariant is defined as

$$
s(K)=s_{\min }(K)+s_{\max }(K) .
$$

Therefore, $s(K)$ is an even integer, and Rasmussen showed that $s(K)$ depends only on the concordance class of $K$. 


\section{Spanning tREe COMPLEXES}

3.1. Construction of Tait's checkerboard graph. Let $D$ be a diagram of a knot $K$. Color regions of $D$ white and black in a checkerboard fashion, i.e. so that if two regions are separated by an $\operatorname{arc}$ of $D$, then they are different colors. The checkerboard coloring gives rise to the two Tait checkerboard graphs $G$ and $G^{*}$ of $D$. The vertices of $G$ are in one-to-one correspondence with the black regions, and the edges of $G$ are in one-to-one correspondence with the crossings of $D$. Each edge in $G$ is incident to the vertices that correspond to the black regions near the crossing. An edge in $G$ is called an $A$-edge (respectively a $B$-edge) if the $A$ smoothing (respectively the $B$-smoothing) separates the black regions. The vertices of $G^{*}$ are in one-to-one correspondence with the white regions, and the edges of $G^{*}$ are in one-to-one correspondence with the crossings of $D$. Each edge in $G^{*}$ is incident to the vertices that correspond to the white regions near the crossing. If an edge in $G$ is an $A$-edge (respectively a $B$-edge), then the edge corresponding to the same crossing in $G^{*}$ is a $B$-edge (respectively an $A$-edge). Observe that $G^{*}$ is the planar dual of $G$. We choose the checkerboard coloring so that the number of $B$-edges in $G$ is greater than or equal to the number of $B$-edges in $G^{*}$. Figure 3 shows an example of the Tait graphs for the $10_{124}$ knot. Let $\mathcal{T}(G)$ denote the set of spanning trees of $G$.
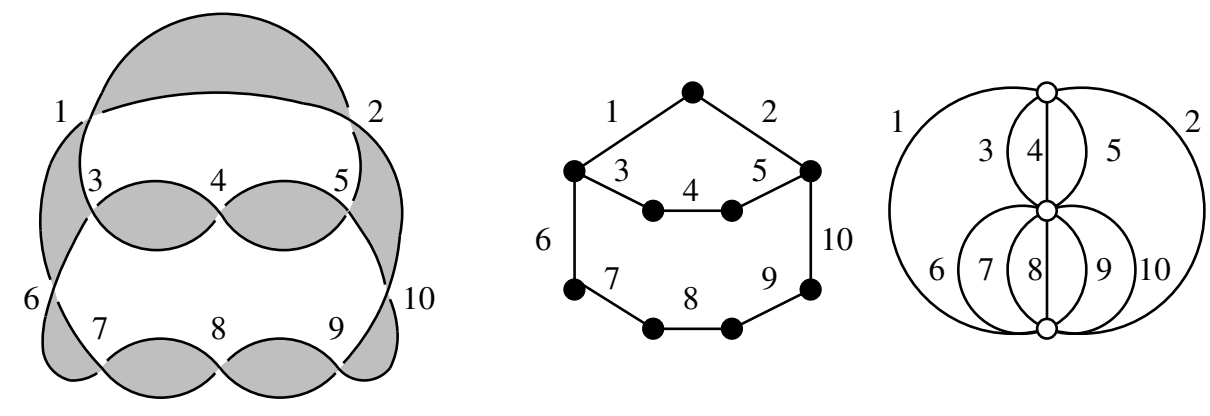

FiguRE 3. A diagram of the $10_{124}$ knot, along with its two Tait graphs. In the black graph (center), edges 1 and 2 are $A$-edges, while edges 3 through 10 are $B$-edges. Conversely, in the white graph (right), edges 1 and 2 are $B$-edges, while edges 3 through 10 are $A$-edges.

For any subgraph $H$ of $G$, let $V(H)$ be the number of vertices in $H$. Each edge in $G$ is associated to a crossing of $D$, and each crossing in $D$ is either positive or negative (see Figure 2). Moreover, each edge in $G$ is either an $A$-edge or a $B$-edge. For any subgraph $H$ of $G$ or $G^{*}$, let $E_{A}^{+}(H)$ denote the number of edges in $H$ that are both $A$-edges and associated to a positive crossing. Similarly define $E_{A}^{-}(H)$, $E_{B}^{+}(H)$, and $E_{B}^{-}(H)$. Also, let $E^{+}(H)$ denote the number of edges in $H$ associated to positive crossings in $D$ and let $E^{-}(H)$ denote the number of edges in $H$ associated to negative crossings in $D$. Note that $E^{+}(D)=n_{+}(D)$ and $E^{-}(G)=n_{-}(D)$. Since many of the subsequent arguments rely on graph-theoretic ideas, we favor using $E^{ \pm}(G)$ over $n_{ \pm}(D)$. Similarly, let $E_{A}(H)$ be the number of $A$-edges in $H$ and let $E_{B}(H)$ be the number of $B$-edges in $H$. We alert the reader that in the literature $A$-edges are sometimes called negative edges and $B$-edges are called positive edges. 
Since we have a different notion of positive and negative edges, we use the $A$ and $B$ notation instead.

If $M=\bigoplus M_{i, j}$ is a finitely generated, bigraded $\mathbb{Z}$-module, then define the $\delta$ grading of $M$ by $\delta=j-i$.

3.2. The knot Floer homology spanning tree complex. In OS03a, Ozsváth and Szabó showed how to associate a Heegaard diagram $(\Sigma, \alpha, \beta, w, z)$ to a knot diagram $D$ such that the intersection points of the tori $\mathbb{T}_{\alpha}$ and $\mathbb{T}_{\beta}$ embedded into $\operatorname{Sym}^{g}(\Sigma)$ are in one-to-one correspondence with the spanning trees of the Tait graph of $D$. Hence there exists a complex whose homology is knot Floer homology that is generated by the spanning trees of the Tait graph.

Proposition 3.1 (Ozsváth, Szabó OS03a). Let $D$ be a diagram of a knot $K$ and let $G$ be its Tait graph. There exists a complex $\widehat{C F K}(D)$ whose generators are in one-to-one correspondence with the spanning trees of $G$ and whose homology is $\widehat{H F K}(K)$.

Ozsváth and Szabó OS03a showed how to calculate the $\delta$-grading of a generator by taking a certain sum over the crossings of the knot diagram. In [Low08, the second author interpreted the $\delta$-grading in terms of information about the Tait graph of the knot diagram. The $\delta$-grading corresponding to a spanning tree $T$ is

$$
\delta_{\widehat{H F K}}(T)=\frac{1}{2}\left(E_{B}^{+}(T)+E_{A}^{+}(G \backslash T)-E_{A}^{-}(T)-E_{B}^{-}(G \backslash T)\right) .
$$

3.3. The Khovanov homology spanning tree complex. In the cube of resolutions complex for Khovanov homology $C K h(D)$, one associates a two-dimensional vector space to each connected component of a Kauffman state. Wehrli Weh08 and Champanerkar and Kofman CK09] showed that the cube of resolutions $C K h(D)$ retracts onto a complex where one associates a two-dimensional vector space to each partial resolution of the knot diagram $D$ that is a twisted unknot (a partial resolution of $D$ that can be transformed into the trivial diagram of the unknot via Reidemeister one moves). The partial resolutions of $D$ that are twisted unknots are in one-to-one correspondence with the spanning trees of the Tait graph of $D$. Similarly, there is a spanning tree complex for reduced Khovanov homology.

Let $G$ be the Tait graph of a knot diagram $D$, and let $\mathcal{T}(G)$ the set of spanning trees of $G$. Define the spanning tree complex for Khovanov homology as

$$
C(D)=\bigoplus_{T \in \mathcal{T}(G)} \mathbb{Z}\left[T_{+}, T_{-}\right]
$$

and define the spanning tree complex for reduced Khovanov homology as

$$
\widetilde{C}(D)=\bigoplus_{T \in \mathcal{T}(G)} \mathbb{Z}[T]
$$

Proposition 3.2 (Wehrli Weh08], Champanerkar-Kofman [CK09]). Let D be a diagram of a knot $K$.

(1) There exists a spanning tree complex $C(D)$ whose homology is $K h(K)$.

(2) There exists a spanning tree complex $\widetilde{C}(D)$ whose homology is $\widetilde{K h}(K)$.

Champanerkar and Kofman chose their gradings so that the bigraded Euler characteristic of $\widetilde{K h}(K)$ is $q^{-1} V_{K}\left(q^{2}\right)$ where $V_{K}(q)$ is the Jones polynomial of $K$. We replace their $j$-grading by $\frac{j+1}{2}$ so that the bigraded Euler characteristic is $V_{K}(q)$. 
The gradings between the Khovanov complex and the reduced Khovanov complex are related by

$$
\begin{gathered}
i_{K h}\left(T_{+}\right)=i_{\widetilde{K h}}(T)=i_{K h}\left(T_{-}\right) \text {and } \\
j_{K h}\left(T_{+}\right)-\frac{1}{2}=j_{\widetilde{K h}}(T)=j_{K h}\left(T_{-}\right)+\frac{1}{2},
\end{gathered}
$$

for any tree $T \in \mathcal{T}(G)$. The $\delta$-grading corresponding to a spanning tree $T$ in $\widetilde{C}(D)$ is

$$
\delta_{\widetilde{K h}}(T)=E_{B}(T)+\frac{1}{4}\left(E^{+}(G)-E^{-}(G)-E_{B}(G)+E_{A}(G)-2(V(G)-1)\right) .
$$

For our convenience, we give two alternate formulations of $\delta_{\overparen{K h}}(T)$. Since $T$ is a spanning tree, $V(G)-1=E(T)=E_{A}(T)+E_{B}(T)$, and thus

$$
\begin{aligned}
2 \delta_{\overparen{K h}}(T) & =2 E_{B}(T)+\frac{1}{2}\left(E^{+}(G)-E^{-}(G)-E_{B}(G)+E_{A}(G)-2\left(E_{A}(T)+E_{B}(T)\right)\right) \\
& =E_{B}(T)-E_{A}(T)+\frac{1}{2}\left(E^{+}(G)-E^{-}(G)-E_{B}(G)+E_{A}(G)\right) .
\end{aligned}
$$

The number of crossings of $D$ can be counted in two ways: by counting positive and negative crossings in $D$ and by counting $A$-edges and $B$-edges in $G$. Therefore, $E^{+}(G)+E^{-}(G)=E_{A}(G)+E_{B}(G)$ or said another way $E^{+}(G)-E_{B}(G)=E_{A}(G)-$ $E^{-}(G)$. This leads to our two new formulations of $\delta_{K h}(T)$ :

$$
\begin{aligned}
& 2 \delta_{\widetilde{K h}}(T)=E_{B}(T)-E_{A}(T)+E^{+}(G)-E_{B}(G), \text { and } \\
& 2 \delta_{\widetilde{K h}}(T)=E_{B}(T)-E_{A}(T)-E^{-}(G)+E_{A}(G) .
\end{aligned}
$$

3.4. The $\delta$-grading. The $\delta$-grading of a spanning tree when considered in the reduced Khovanov complex is the same as the $\delta$-grading of that spanning tree when considered in the knot Floer complex. We note that this is not true of either the homological or polynomial (Jones or Alexander) gradings individually.

Proposition 3.3. Let $G$ be the Tait graph of a knot diagram D. If $T$ is a spanning tree of $G$, then $\delta_{\overparen{K h}}(T)=\delta_{\widehat{H F K}}(T)$.

Proof. From equation (3.1), we have

$$
\begin{aligned}
2 \delta_{\overparen{K h}}(T) & =E_{B}(T)-E_{A}(T)+E^{+}(G)-E_{B}(G) \\
& =E_{B}^{+}(T)+E_{B}^{-}(T)-E_{A}^{+}(T)-E_{A}^{-}(T)+E_{A}^{+}(G)+E_{B}^{+}(G)-E_{B}^{+}(G)-E_{B}^{-}(G) \\
& =E_{B}^{+}(T)+E_{B}^{-}(T)-E_{A}^{+}(T)-E_{A}^{-}(T)+E_{A}^{+}(G)-E_{B}^{-}(G) \\
& =E_{B}^{+}(T)-E_{B}^{-}(G \backslash T)-E_{A}^{-}(T)+E_{A}^{+}(G \backslash T) \\
& =2 \delta_{\widehat{H F K}}(T) .
\end{aligned}
$$

For the remainder of the paper, we use the notation $\delta(T)$ to equivalently mean $\delta_{\overparen{K h}}(T)$ or $\delta_{\overparen{H F K}}(T)$. Define

$$
\delta_{\min }(D)=\min \{\delta(T) \mid T \in \mathcal{T}(G)\} \text { and } \delta_{\max }(D)=\max \{\delta(T) \mid T \in \mathcal{T}(G)\} .
$$

Proposition 3.4. Let $D$ be a diagram of a knot $K$. Then $\delta_{\min }(D) \leq \tau(K) \leq$ $\delta_{\max }(D)$. 
Proof. Proposition 3.1 implies there is a Heegaard diagram subordinate to $K$ where the intersections points of $\mathbb{T}_{\alpha}$ and $\mathbb{T}_{\beta}$ are in one-to-one correspondence with the spanning trees of the Tait graph $G$. One can use this Heegaard diagram to generate both the complexes $\widehat{C F}\left(S^{3}\right)$ and $\widehat{C F K}(K)$. By the definition of $\tau$, there must be some spanning tree $T$ in filtration level $\tau$. Since the generator of $\widehat{H F}\left(S^{3}\right)$ is in homological grading 0 , the tree $T$ must also be in homological grading 0 . Therefore, the tree $T$ (viewed as a generator of $\widehat{C F K}(K))$ must satisfy $\delta(T)=\tau(K)$.

Proposition 3.5. Let $D$ be a diagram of a knot $K$. Then $2 \delta_{\min }(D) \leq s(K) \leq$ $2 \delta_{\max }(D)$.

Proof. Since $C(D)$ is a deformation retract of $C K h(D)$, there exists a spectral sequence (analogous to the sequence of Theorem 2.1) whose $E_{1}$ page is $C(D)$, whose $E_{2}$ page is $K h(K)$ and which converges to $\mathbb{Q} \oplus \mathbb{Q}$. Therefore, there exist two generators $T_{1}$ and $T_{2}$ of $C(D)$ with $i_{K h}\left(T_{1}\right)=i_{K h}\left(T_{2}\right)=0$ and $j_{K h}\left(T_{1}\right)=s_{\min }(K)$ and $j_{K h}\left(T_{2}\right)=s_{\max }(K)$. Hence, there exists a spanning tree $T$ such that $\delta_{\overline{K h}}(T)=$ $s(K) / 2$.

\section{The Turaev surface}

The ideas discussed below involve ribbon graphs associated to a knot diagram. These ideas are developed by Dasbach, Futer, Kalfagianni, Lin, and Stoltzfus (cf. $\mathrm{DFK}^{+} 06$ and $\left.\mathrm{DFK}^{+} 08\right]$. The construction of the Turaev surface of a knot diagram is due to Turaev [Tur87.

Let $D$ be a knot diagram and let $\Gamma$ be the 4 -valent plane graph obtained from $D$ by forgetting the "over-under" information at each crossing. Regard $\Gamma$ as embedded in $\mathbb{R}^{2}$ which is sitting inside $\mathbb{R}^{3}$. Remove a neighborhood around each vertex of $\Gamma$, resulting in a collection of arcs in the plane. Replace each arc by a band which is perpendicular to the plane. In the neighborhoods removed earlier, place a saddle so that the circles obtained from choosing an $A$ resolution at each crossing lie above the plane and so that the circles obtained from choosing a $B$ resolution at each crossing lie below the plane. Such a saddle is shown in Figure 4 . The boundary of

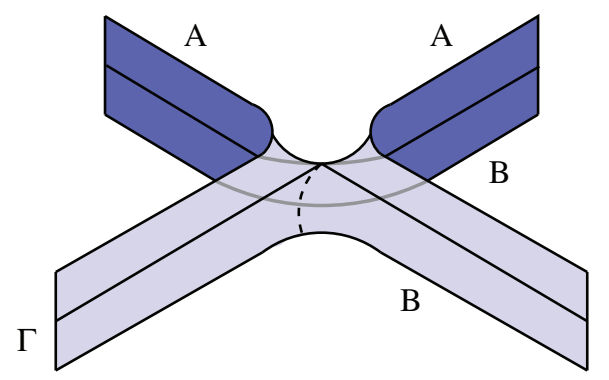

FiguRE 4. In a neighborhood of each vertex of $\Gamma$ a saddle surface transitions between the $A$ and $B$ circles.

the resulting surface is a collection of disjoint circles, where circles corresponding to the all- $A$ resolution lie above the plane and circles corresponding to the all- $B$ resolution lie below the plane. Cap off each boundary circle with a disk to obtain $\Sigma_{D}$, the Turaev surface of $D$. The Turaev genus of a knot $K$ is defined as

$$
g_{T}(K)=\min \left\{g\left(\Sigma_{D}\right) \mid D \text { is a diagram of } K\right\} .
$$


A ribbon graph is a graph together with a cellular embedding into a surface. The genus $g(\mathbb{G})$ of a ribbon graph is the genus of the surface into which it embeds. Denote the number of vertices in a ribbon graph $\mathbb{G}$ by $V(\mathbb{G})$. One can embed two ribbon graphs $\mathbb{A}$ and $\mathbb{B}$ into $\Sigma_{D}$ as follows. The vertices of $\mathbb{A}$ correspond to the disks used to cap off the $A$ circles, and the edges of $\mathbb{A}$ are the flowlines going from the vertices through the saddles. Similarly, the vertices of $\mathbb{B}$ correspond to the disks used to cap off the $B$ circles, and the edges of $\mathbb{B}$ are the flowlines going from the vertices through the saddles. The ribbon graphs $\mathbb{A}$ and $\mathbb{B}$ are dual to one another on $\Sigma_{D}$, and therefore the Euler characteristic of $\Sigma_{D}$ is determined by

$$
\chi\left(\Sigma_{D}\right)=s_{A}(D)-c(D)+s_{B}(D),
$$

where $c(D)$ is the number of crossings of $D$ and $s_{A}(D)$ and $s_{B}(D)$ are the number of components in the all- $A$ smoothing and all- $B$ smoothing respectively.

Let $\mathbb{G}$ be a ribbon graph. A ribbon subgraph $\mathbb{H}$ of $\mathbb{G}$ is a subgraph of $\mathbb{G}$ such that the cyclic orientation of the edges in the embedding of $\mathbb{H}$ is inherited from the embedding of $\mathbb{G}$. Note that the surfaces on which $\mathbb{H}$ and $\mathbb{G}$ are embedded are not necessarily the same. If $\mathbb{G}$ is embedded on the surface $\Sigma$, then the connected components of $\Sigma \backslash \mathbb{G}$ are known as the faces of $\mathbb{G}$. A spanning quasi-tree $\mathbb{T}$ of $\mathbb{G}$ is a connected ribbon subgraph of $\mathbb{G}$ such that $V(\mathbb{T})=V(\mathbb{G})$ and such that $\mathbb{T}$ has one face. Denote the set of spanning quasi-trees of $\mathbb{G}$ by $Q(\mathbb{G})$.

Recall that $\mathcal{T}(G)$ denotes the set of spanning trees of the Tait graph $G$. Champanerkar, Kofman, and Stoltzfus CKS07 defined maps $q_{\mathbb{A}}: \mathcal{T}(G) \rightarrow \mathcal{Q}(\mathbb{A})$ and $q_{\mathbb{B}}: \mathcal{T}(G) \rightarrow \mathcal{Q}(\mathbb{B})$. Since the sets of edges of $G, \mathbb{A}$, and $\mathbb{B}$ are each in one-to-one correspondence with the crossings of $D$, we identify all three sets. Because elements of $\mathcal{T}(G), \mathcal{Q}(\mathbb{A})$, and $\mathcal{Q}(\mathbb{B})$ are spanning, it suffices to define $q_{\mathbb{A}}$ and $q_{\mathbb{B}}$ on the set of edges of $G$. Let $T$ be a spanning tree of $G$. An $A$-edge of $G$ is in the quasi-tree $q_{\mathbb{A}}(T)$ if and only if it is in $T$, and a $B$-edge of $G$ is in the quasi-tree $q_{\mathbb{A}}(T)$ if and only if it is in $G \backslash T$. Similarly, an $A$-edge of $G$ is in the quasi-tree $q_{\mathbb{B}}(T)$ if and only if it is in $G \backslash T$, and a $B$-edge of $G$ is in $q_{\mathbb{B}}(T)$ if and only if it is in $T$.

Theorem 4.1 (Champanerkar, Kofman, Stoltzfus [CKS07]). The maps

$$
q_{\mathbb{A}}: \mathcal{T}(G) \rightarrow \mathcal{Q}(\mathbb{A}) \text { and } q_{\mathbb{B}}: \mathcal{T}(G) \rightarrow \mathcal{Q}(\mathbb{B})
$$

are bijections. Moreover, the genera of $q_{\mathbb{A}}(T)$ and $q_{\mathbb{B}}(T)$ are determined by

$$
\begin{aligned}
g\left(q_{\mathbb{A}}(T)\right)+E_{B}(T) & =\frac{V(G)+E_{B}(G)-s_{A}(D)}{2} \text { and } \\
g\left(q_{\mathbb{B}}(T)\right)+E_{A}(T) & =\frac{V(G)+E_{A}(G)-s_{B}(D)}{2} .
\end{aligned}
$$

In the spanning tree complex for reduced Khovanov homology, the $\delta$-grading for each spanning tree $T$ is the number of $B$-edges in $T$ (up to some overall shift dependent on the diagram $D$ ). Champanerkar, Kofman, and Stoltzfus [CKS07 use Theorem 4.1 to show that for any knot diagram, the difference between the maximum and minimum $\delta$-gradings in the spanning tree complex for reduced Khovanov homology is equal to the genus of the Turaev surface of that diagram. Using a different approach, the second author [Low08] proved that for any knot diagram, the difference between the maximum and minimum $\delta$-gradings in the spanning tree complex for knot Floer homology is equal to the genus of the Turaev surface of that diagram. In light of Proposition 3.3. the second author's result can be shown 
using the same approach as Champanerkar, Kofman, and Stoltzfus [CKS07, and both results can be encoded by the equation

$$
g\left(\Sigma_{D}\right)=\delta_{\max }(D)-\delta_{\min }(D),
$$

where $D$ is a knot diagram and $\Sigma_{D}$ is the Turaev surface of $D$.

The maximum and minimum $\delta$-gradings are related to Traczyk's combinatorial data coming from a diagram of the knot.

Corollary 4.2. Let $D$ be a knot diagram, and let $G$ be its Tait graph. Then

$$
\begin{aligned}
2 \delta_{\min }(D) & =s_{B}(D)-E^{-}(G)-1 \text { and } \\
2 \delta_{\max }(D) & =1+E^{+}(G)-s_{A}(D) .
\end{aligned}
$$

Proof. Let $T_{\min }$ be a spanning tree such that $\delta\left(T_{\min }\right)=\delta_{\min }(D)$. By the definition of $q_{\mathbb{B}}$, the number of edges in $q_{\mathbb{B}}\left(T_{\min }\right)$ is $E_{A}\left(G \backslash T_{\min }\right)+E_{B}\left(T_{\min }\right)$. Since $\delta\left(T_{\min }\right)=$ $\delta_{\min }(D)$, the tree $T_{\min }$ has the maximum number of $A$-edges possible, and thus Theorem 4.1 implies that $g\left(q_{\mathbb{B}}\left(T_{\min }\right)\right)=0$. Therefore, $q_{\mathbb{B}}\left(T_{\min }\right)$ is a spanning tree of the underlying graph of $\mathbb{B}$ and has $s_{B}(D)-1$ edges.

Equation (3.1) implies

$$
\begin{aligned}
2 \delta\left(T_{\min }\right) & =E_{B}\left(T_{\min }\right)-E_{A}\left(T_{\min }\right)+E_{A}(G)-E^{-}(G) \\
& =E_{A}\left(G \backslash T_{\min }\right)+E_{B}\left(T_{\min }\right)-E^{-}(G) \\
& =s_{B}(D)-E^{-}(G)-1 .
\end{aligned}
$$

Similarly, let $T_{\max }$ be a spanning tree such that $\delta\left(T_{\max }\right)=\delta_{\max }(D)$. By the definition of $q_{\mathbb{A}}$, the number of edges in $q_{\mathbb{A}}\left(T_{\max }\right)$ is $E_{A}\left(T_{\max }\right)+E_{B}\left(G \backslash T_{\max }\right)$. Since $\delta\left(T_{\max }\right)=\delta_{\max }(D)$, the tree $T_{\max }$ has the maximum number of $B$-edges possible, and thus Theorem 4.1 implies that $g\left(q_{\mathbb{A}}\left(T_{\max }\right)\right)=0$. Therefore, $q_{\mathbb{A}}\left(T_{\max }\right)$ is a spanning tree of the underlying graph of $\mathbb{A}$ and has $s_{A}(D)-1$ edges.

Equation (3.2) implies

$$
\begin{aligned}
2 \delta\left(T_{\max }\right) & =E_{B}\left(T_{\max }\right)-E_{A}\left(T_{\max }\right)+E^{+}(G)-E_{B}(G) \\
& =E^{+}(G)-E_{B}\left(G \backslash T_{\max }\right)-E_{A}\left(T_{\max }\right) \\
& =1+E^{+}(G)-s_{A}(D) .
\end{aligned}
$$

\section{Knot Signature}

The signature of a knot $\sigma(K)$ was defined by Trotter in Tro62 and was shown to be a concordance invariant by Murasugi in [Mur65] (see also [KT76]). In this section, we show that $\sigma(K)$ satisfies inequalities similar to the inequalities satisfied by $\tau(K)$ and $s(K)$. Consequently, one has new lower bounds for the Turaev genus of a knot.

Let $G$ be the Tait graph of a diagram $D$ of a knot $K$. Define $G_{A}$ and $G_{B}$ to be the graphs obtained from $G$ by deleting all $B$-edges and $A$-edges, respectively. Furthermore, for any graph $\Gamma$ define $\beta_{0}(\Gamma)$ and $\beta_{1}(\Gamma)$ to be the zeroth and first Betti numbers of $\Gamma$ (thought of as a 1-complex).

One can use results of Murasugi Mur89] and Thistlethwaite Thi88 to obtain the desired inequality for knot signature. We warn the reader that the previous two references have opposite conventions for labeling edges of $G$ positive or negative. Translated into the notation of this paper, equation (13.4) of Murasugi [Mur89] is the following proposition. 
Proposition 5.1 (Murasugi [Mur89]). Let $G$ be the Tait graph of a diagram of the knot $K$. Then

$$
\beta_{0}\left(G_{B}\right)+\beta_{1}\left(G_{B}\right)-E^{+}(G)-1 \leq \sigma(K) \leq E^{-}(G)-\beta_{0}\left(G_{A}\right)-\beta_{1}\left(G_{A}\right)+1 .
$$

Also, the following proposition appears in Thistlethwaite [Thi88].

Proposition 5.2 (Thistlethwaite Thi88]). Let $G$ be the Tait graph of a knot diagram D. Then

$$
\begin{aligned}
& s_{A}(D)=\beta_{0}\left(G_{B}\right)+\beta_{1}\left(G_{B}\right) \text { and } \\
& s_{B}(D)=\beta_{0}\left(G_{A}\right)+\beta_{1}\left(G_{A}\right) .
\end{aligned}
$$

Propositions 5.1 and 5.2 imply the following result.

Proposition 5.3. Let $D$ be a diagram of a knot $K$. Then $2 \delta_{\min }(D) \leq-\sigma(K) \leq$ $2 \delta_{\max }(D)$.

Proof. We have

$$
\begin{aligned}
2 \delta_{\min }(D) & =s_{B}(D)-E^{-}(G)-1 \\
& =\beta_{0}\left(G_{A}\right)+\beta_{1}\left(G_{A}\right)-E^{-}(G)-1 \\
& \leq-\sigma(K)
\end{aligned}
$$

Similarly,

$$
\begin{aligned}
2 \delta_{\max }(D) & =1+E^{+}(G)-s_{A}(D) \\
& =1+E^{+}(G)-\beta_{0}\left(G_{B}\right)-\beta_{1}\left(G_{B}\right) \\
& \geq-\sigma(K) .
\end{aligned}
$$

Proof of Theorem 1.1. Propositions 3.4 3.5, and 5.3 give the following inequalities:

$$
\begin{aligned}
2 \delta_{\min }(D) \leq 2 \tau(K) & \leq 2 \delta_{\max }(D), \\
2 \delta_{\min }(D) \leq s(K) & \leq 2 \delta_{\max }(D), \text { and } \\
2 \delta_{\min }(D) \leq-\sigma(K) & \leq 2 \delta_{\max }(D) .
\end{aligned}
$$

The result now follows from equation (4.1).

Lobb Lob09] gave upper and lower bounds on the Rasmussen $s$ invariant. Lobb's bounds also depend on the diagram of the knot. He used combinatorial data obtained from the oriented resolution of the diagram. Our results are similar in nature, but we use combinatorial data obtained from the all- $A$ and all- $B$ resolutions.

\section{EXAmPle: 3-BRAID KNOTS}

In this section, we examine knots obtained as the closure of a 3-braid and compute the bounds of Theorem 1.1 for each such knot. In many cases, this leads to a computation of the Turaev genus of such knots.

Let $B_{3}$ denote the braid group on three strands, generated by elements $\sigma_{1}$ and $\sigma_{2}$. Murasugi described the conjugacy classes of closed 3-braids.

Theorem 6.1 (Murasugi Mur74). Any 3-braid is conjugate to exactly one braid of the form $\left(\sigma_{1} \sigma_{2}\right)^{3 n} \cdot w$, where $n \in \mathbb{Z}$ and $w$ is either

(1) equal to $\sigma_{1}^{a_{1}} \sigma_{2}^{-b_{1}} \cdots \sigma_{1}^{a_{k}} \sigma_{2}^{-b_{k}}$, where $a_{i}, b_{i}>0$;

(2) equal to $\sigma_{2}^{k}$ for some $k \in \mathbb{Z}$; or

(3) equal to $\sigma_{1}^{m} \sigma_{2}^{-1}$ where $m \in\{-1,-2,-3\}$. 
We say a 3-braid in one of the above forms is in Murasugi normal form. Closed 3 -braids whose Murasugi normal form is of type (2) or type (3) with $m=-2$ are links. A closed 3-braid knot of type (3) is a $(3, q)$ torus knot.

6.1. Torus knots. Let $T(3, q)$ denote the $(3, q)$ torus knot. Throughout this subsection, we assume $q>0$. The computations for $q<0$ are similar. Ozsváth and Szabó OS03b and Rasmussen Ras04 computed the value of the $\tau$ and $s$ invariants for torus knots. In our case, we have

$$
2 \tau(T(3, q))=s(T(3, q))=2 q-2 .
$$

Gordon, Litherland, and Murasugi GLM81 showed that the signature of a $(3, q)$ torus knot is given by

$$
\sigma(T(3,3 k+l))= \begin{cases}-4 k-2 l+2 & \text { if } k \text { is even, } \\ -4 k-2 l & \text { if } k \text { is odd, }\end{cases}
$$

for $l=1$ or 2 . If $k$ is even, the bounds from Theorem 1.1 are

$$
\begin{aligned}
\mid \tau(T & (3,3 k+l))+\frac{\sigma(T(3,3 k+l))}{2} \mid \\
& =\left|\frac{s(T(3,3 k+l))+\sigma(T(3,3 k+l))}{2}\right|=k,
\end{aligned}
$$

for $l=1$ or 2 . If $k$ is odd, the bounds from Theorem 1.1 are

$$
\begin{aligned}
& \left|\tau(T(3,3 k+l))+\frac{\sigma(T(3,3 k+l))}{2}\right| \\
& \quad=\left|\frac{s(T(3,3 k+l))+\sigma(T(3,3 k+l))}{2}\right|=k-1,
\end{aligned}
$$

for $l=1$ or 2 .

In Low09], the second author found knot diagrams $D_{k, l}$ of the knots $T(3,3 k+l)$ such that the genus of the Turaev surface is given by

$$
g\left(\Sigma_{D_{k, l}}\right)=k,
$$

where $l=1$ or 2 . Therefore equations (6.1) and (6.2) and Theorem 1.1 imply that

$$
g_{T}(T(3,3 k+l))=k,
$$

for $k$ even and $l=1$ or 2 , and

$$
g_{T}(T(3,3 k+l))=k \text { or } k-1,
$$

for $k$ odd and $l=1$ or 2 . However, using the lower bounds on Turaev genus given by reduced Khovanov width [CKS07, the second author [Low09] has shown that

$$
g_{T}(T(3,3 k+l))=k,
$$

for all $k>0$ and $l=1$ or 2 . Thus, if $k$ is odd, then the bounds of Theorem 1.1 for $T(3,3 k+l)$ are strictly less than the Turaev genus. 
6.2. Non-torus closed 3-braids. We now turn our attention to closed 3-braid knots whose Murasugi normal form is of type (1). Throughout this subsection, we assume $n>0$. The computations when $n<0$ are similar.

Proposition 6.2. Let $K_{n}$ be the closure of $\left(\sigma_{1} \sigma_{2}\right)^{3 n} \sigma_{1}^{a_{1}} \sigma_{2}^{-b_{1}} \cdots \sigma_{1}^{a_{k}} \sigma_{2}^{-b_{k}}$. Then

$$
g_{T}(K)=n \text { or } n-1 \text {. }
$$

We will prove Proposition 6.2 using the bounds from Theorem 1.1 and so it is first necessary to compute $\tau\left(K_{n}\right), s\left(K_{n}\right)$, and $\sigma\left(K_{n}\right)$. Erle calculated the signature of such a closed 3-braid knot.

Proposition 6.3 (Erle Erl99]). If $K_{n}$ is the closure of $\left(\sigma_{1} \sigma_{2}\right)^{3 n} \sigma_{1}^{a_{1}} \sigma_{2}^{-b_{1}} \cdots \sigma_{1}^{a_{k}} \sigma_{2}^{-b_{k}}$, then

$$
\sigma\left(K_{n}\right)=-4 n-\sum_{i=1}^{k}\left(a_{i}-b_{i}\right)
$$

Using work of Van Cott Cot08, Greene computed the Rasmussen $s$ invariant for such closed 3-braids.

Proposition 6.4 (Greene Gre09). Let $K_{n}$ be a knot that is the closure of $\left(\sigma_{1} \sigma_{2}\right)^{3 n} \sigma_{1}^{a_{1}} \sigma_{2}^{-b_{1}} \cdots \sigma_{1}^{a_{k}} \sigma_{2}^{-b_{k}}$. Then

$$
s\left(K_{n}\right)= \begin{cases}6 n-2-\sigma\left(K_{0}\right), & \text { if } n>0 ; \\ -\sigma\left(K_{0}\right), & \text { if } n=0 ; \\ 6 n+2-\sigma\left(K_{0}\right), & \text { if } n<0 .\end{cases}
$$

Greene's proof depends on the following facts:

(1) For a quasi-alternating knot $s(K)=-\sigma(K)$.

(2) $s$ is a homomorphism from the smooth knot concordance group $\mathcal{C} \rightarrow \mathbb{Z}$.

(3) $|s(K)| \leq 2 g_{4}(K)$, where $g_{4}(K)$ is the 4-genus of $K$.

(4) $s$ of the $(m, n)$ torus knot is $(m-1)(n-1)$.

Note that $(2)-(4)$ above are the conditions appearing in Van Cott's Cot08] work.

Each of $(1)-(4)$ also holds for $2 \tau$, and so, using the notation of Proposition 6.4. we have

$$
2 \tau\left(K_{n}\right)=s\left(K_{n}\right) .
$$

Proof of Proposition 6.2. The bounds of Theorem 1.1 are given by

$$
\left|\frac{s\left(K_{n}\right)+\sigma\left(K_{n}\right)}{2}\right|=\left|\tau\left(K_{n}\right)+\frac{\sigma\left(K_{n}\right)}{2}\right|=n-1 .
$$

The second author [Low09] showed that $g_{T}\left(K_{n}\right) \leq n$. Hence

$$
g_{T}\left(K_{n}\right)=n-1 \text { or } n \text {. }
$$

\section{ACKnowledgments}

The authors would like to thank Josh Greene for helpful conversations. Also, the authors would like to thank the referee, whose suggestions significantly improved the exposition in this paper. 


\section{REFERENCES}

[Abe09] Tetsuya Abe, An estimation of the alternation number of a torus knot, J. Knot Theory Ramifications 18 (2009), no. 3, 363-379. MR2514849 (2010b:57004)

[CK09] Abhijit Champanerkar and Ilya Kofman, Spanning trees and Khovanov homology, Proc. Amer. Math. Soc. 137 (2009), no. 6, 2157-2167. arXiv:math.GT/0607510. MR 2480298 (2009m:57019)

[CKS07] Abhijit Champanerkar, Ilya Kofman, and Neal Stoltzfus, Graphs on surfaces and Khovanov homology, Algebr. Geom. Topol. 7 (2007), 1531-1540, arXiv:math/0705.3453. MR.2366169 (2008m:57005)

[Cot08] Cornelia Van Cott, Ozsváth-Szabó and Rasmussen invariants of cable knots, Algebr. Geom. Topol. 10 (2010), no. 2, 825-836, arXiv:0803.0500. MR2629765

$\left[\mathrm{DFK}^{+} 06\right]$ Oliver T. Dasbach, David Futer, Efstratia Kalfagianni, Xiao-Song Lin, and Neal W. Stoltzfus, Alternating sum formulae for the determinant and other link invariants, J. Knot Theory Ramifications 19 (2010), no. 6, 765-782, arXiv:math/0611025.

$\left[\mathrm{DFK}^{+} 08\right]$ Oliver T. Dasbach, David Futer, Efstratia Kalfagianni, Xiao-Song Lin, and Neal W. Stoltzfus, The Jones polynomial and graphs on surfaces, J. Combin. Theory Ser. B 98 (2008), no. 2, 384-399, arXiv:math.GT/0605571v3. MR2389605 (2009d:57020)

[Er199] Dieter Erle, Calculation of the signature of a 3-braid link, Kobe J. Math. 16 (1999), no. 2, 161-175. MR.1745024 (2000m:57008)

[GLM81] Cameron McA. Gordon, Richard A. Litherland, and Kunio Murasugi, Signatures of covering links, Canad. J. Math. 33 (1981), no. 2, 381-394. MR617628 (83a:57006)

[Gre09] Joshua Greene, On closed 3-braids with unknotting number one, arXiv:0902.1573., 2009.

[HO08] Matthew Hedden and Philip Ording, The Ozsváth-Szabó and Rasmussen concordance invariants are not equal, Amer. J. Math. 130 (2008), no. 2, 441-453, arXiv:math/0512348. MR2405163 (2009b:57021)

[Kho00] Mikhail Khovanov, A categorification of the Jones polynomial, Duke Math. J. 101 (2000), no. 3, 359-426. MR1740682

[KT76] Louis H. Kauffman and Laurence R. Taylor, Signature of links, Trans. Amer. Math. Soc. 216 (1976), 351-365. MR0388373 (52:9210)

[Lee05] Eun Soo Lee, An endomorphism of the Khovanov invariant, Adv. Math. 197 (2005), no. 2, 554-586, arXiv:math.GT/0210213v3. MR2173845 (2006g:57024)

[Liv04] Charles Livingston, Computations of the Ozsváth-Szabó knot concordance invariant, Geom. Topol. 8 (2004), 735-742 (electronic), arXiv:math/0311036. MR2057779 (2005d:57019)

[Lob09] Andrew Lobb, Computable bounds for Rasmussen's concordance invariant, to appear in Compos. Math., arXiv:0908.2745, 2009.

[Low08] Adam Lowrance, On knot Floer width and Turaev genus, Algebr. Geom. Topol. 8 (2008), no. 2, 1141-1162, arXiv:math.GT/0709.0720v1. MR2443110 (2010f:57036)

[Low09] Adam Lowrance, The Khovanov width of twisted links and closed 3-braids, to appear in Comment. Math. Helv., arXiv:0901.2196, 2009.

[Mur65] Kunio Murasugi, On a certain numerical invariant of link types, Trans. Amer. Math. Soc. 117 (1965), 387-422. MR0171275 (30:1506)

[Mur74] Kunio Murasugi, On closed 3-braids, American Mathematical Society, Providence, R.I., 1974, Memoirs of the American Mathematical Society, No. 151. MR0356023 $(50: 8496)$

[Mur89] Kunio Murasugi, On invariants of graphs with applications to knot theory, Trans. Amer. Math. Soc. 314 (1989), no. 1, 1-49. MR930077 (89k:57016)

[OS03a] Peter Ozsváth and Zoltán Szabó, Heegaard Floer homology and alternating knots, Geom. Topol. 7 (2003), 225-254 (electronic), arXiv:math.GT/0209149. MR.1988285 (2004f:57040)

[OS03b] Peter Ozsváth and Zoltán Szabó, Knot Floer homology and the four-ball genus, Geom. Topol. 7 (2003), 615-639 (electronic), arXiv:math.GT/0301149v4. MR2026543 (2004i:57036)

[OS04a] Peter Ozsváth and Zoltán Szabó, Holomorphic disks and knot invariants, Adv. Math. 186 (2004), no. 1, 58-116. MR2065507 
[OS04b] Peter Ozsváth and Zoltán Szabó, Holomorphic disks and three-manifold invariants: properties and applications, Ann. of Math. (2) 159 (2004), no. 3, 1159-1245. MR.2113020(2006b:57017)

[OS04c] Peter Ozsváth and Zoltán Szabó, Holomorphic disks and topological invariants for closed three-manifolds, Ann. of Math. (2) 159 (2004), no. 3, 1027-1158. MR2113019 (2006b:57016)

[Ras03] Jacob Rasmussen, Floer homology and knot complements, Ph.D. thesis, Harvard University, 2003.

[Ras04] Jacob Rasmussen, Khovanov homology and the slice genus, to appear in Invent. Math., arXiv:math.GT/0402131, 2004.

[Thi88] Morwen B. Thistlethwaite, On the Kauffman polynomial of an adequate link, Invent. Math. 93 (1988), no. 2, 285-296. MR948102 (89g:57009)

[Tra04] Paweł Traczyk, A combinatorial formula for the signature of alternating diagrams, Fund. Math. 184 (2004), 311-316. MR2128055 (2005k:57020)

[Tro62] H. F. Trotter, Homology of group systems with applications to knot theory, Ann. of Math. (2) 76 (1962), 464-498. MR0143201 (26:761)

[Tur87] Vladmir G. Turaev, A simple proof of the Murasugi and Kauffman theorems on alternating links, Enseign. Math. (2) 33 (1987), no. 3-4, 203-225. MR925987 (89e:57002)

[Weh08] Stephan Wehrli, A spanning tree model for Khovanov homology, J. Knot Theory Ramifications 17 (2008), no. 12, 1561-1574, arXiv:math.GT/0409328. MR2477595 (2010d:57016)

Department of Mathematics, Louisiana State University, Baton Rouge, Louisiana 70803-4918

E-mail address: kasten@math.1su.edu

Department of Mathematics, University of Iowa, Iowa City, Iowa 52242-1419

E-mail address: alowrance@math.uiowa.edu 